\title{
Vegetarian diets and risk of diabetes in British adults: results from the EPIC-Oxford study
}

\author{
K. Papier ${ }^{1}$, P. N. Appleby ${ }^{1}$, G.K. Fensom ${ }^{1}$, A. Knuppel ${ }^{1}$, A. Perez-Cornago ${ }^{1}$, J.A. Schmidt ${ }^{1}$, \\ T.Y.N. Tong ${ }^{1}$ and T.J. Key ${ }^{1}$ \\ ${ }^{1}$ Cancer Epidemiology Unit, Nuffield Department of Population Health, University of Oxford, Oxford, OX3 $7 L F$
}

The number of people affected by diabetes globally is rapidly increasing with estimates already surpassing 425 million in 2017 and projected to reach 629 million in $2045^{(1)}$. A few small studies have found that vegetarians might have a lower risk of diabetes than non-vegetarians but they have been limited by small numbers of cases ${ }^{(2,3,4,5)}$. Therefore, we examined the association between vegetarianism and diabetes risk in a large, prospective study of British adults

The analysed cohort included participants from the European Prospective Investigation into Cancer (EPIC)-Oxford study who were diabetes free at recruitment (1993-1999), with available dietary intake data at baseline, and linked hospital admissions and death data for diabetes over follow-up $(1994-2016)(n=45,314)^{(6)}$. Participants were categorized as regular meat eaters $(>50$ grams per day: $n=$ 15,181); low meat eaters ( $<50$ grams of meat per day: $n=7,615)$; fish eaters (ate no meat but consumed fish: $n=7,092)$; and vegetarians and vegans (ate no meat or fish: $\mathrm{n}=15,426$ ). We used multivariable Cox proportional hazards models to assess associations between diet and risk of diabetes.

Over a mean of 17.6 years of follow-up, 1224 incident cases of diabetes were recorded (11 of which were first noted at death). Compared with regular meat eaters, the low meat eaters, fish eaters, and vegetarians and vegans were less likely to develop diabetes (hazard ratio $(\mathrm{HR})=0.63,95 \%$ confidence interval $(\mathrm{CI}) 0.54-0.75$; $\mathrm{HR}=0.47,95 \% \mathrm{CI} 0.38-0.59$; and $\mathrm{HR}=0.63,95 \% \mathrm{CI} 0.54-0.74$, respectively). These associations were substantially attenuated after adjusting for body mass index (BMI) (low meat eaters: HR $=0.78$, $95 \%$ CI 0.66-0.92; fish eaters: $\mathrm{HR}=0.64,95 \%$ CI 0.51-0.80; and vegetarians and vegans: $\mathrm{HR}=0.89,95 \% \mathrm{CI} 0.76-1.05)(\mathrm{Table} 1)$.

Table 1. Associations between diet group and diabetes incidence in 45,314 EPIC-Oxford participants

\begin{tabular}{lccc}
\hline & & \multicolumn{2}{c}{ Hazard ratios, 95\% Confidence intervals } \\
\cline { 2 - 4 } Dietary groups & Cases / total at risk & \multicolumn{1}{c}{ Model 1 } & Model 2 \\
\hline Regular meat eaters & $691 / 15181$ & $0.63(0.54-0.75)$ & 1 \\
Low meat eaters & $184 / 7615$ & $0.47(0.38-0.59)$ & $0.78(0.66-0.92)$ \\
Fish eaters & $93 / 7092$ & $0.63(0.54-0.74)$ & $0.64(0.51-0.80)$ \\
Vegetarians and vegans & $256 / 15426$ & $<0.001$ & $0.89(0.76-1.05)$ \\
$P$-value & - & 0.001 \\
\hline Model 1: Analysis stratified by sex, method of recruitment, region of residence and adjusted for age, education, $^{\text {Townsend deprivation index, ethnicity, smoking, alcohol intake, and physical activity. Model 2: Analysis }}$ \\
further adjusted for BMI. ${ }^{1}$ Represents significant heterogeneity in risk between diet groups based on \\
likelihood-ratio test statistics.
\end{tabular}

We found that consuming a low or meat-free diet was associated with a lower risk of diabetes, and that this was at least partly attributable to these groups having a lower BMI than regular meat eaters. Our findings suggest that consuming low-meat or vegetarian diets may have a role in controlling the growing diabetes epidemic.

This work is supported by Wellcome Trust Our Planet Our Health (Livestock, Environment and People, LEAP 205212/Z/16/Z).

1. International Diabetes Federation (2017) IDF Diabetes Atlas 8th Edition

2. Snowdon DA, Phillips RL (1985) Am J Public Health 75,507-12

3. Chiu THT, Pan WH, Lin MN et al. (2018) Nutr Diabetes 8,12

4. Tonstad S, Stewart K, Oda K et al. (2013) Nutr Metab Cardiovasc Dis 23,292-9

5. Vang A, Singh PN, Lee JW et al. (2008) Ann Nutr Metab 52, 96-104

6. Crowe FL, Appleby PN, Travis RC et al. (2013) Am J Clin Nutr 97, 597-603 